\title{
Outcome of Adjuvant Concurrent Chemo-Radiation in Operated Locally Advanced Rectal Cancer
}

\author{
Syed Arshad Mustafa ${ }^{1}$, M. Ismail ${ }^{2}$, Saquib Zaffar ${ }^{3}$, Ghulam Hassan $^{4}$, \\ Waseem Qureshi ${ }^{5}$, SM Kadri $^{6}$ \\ Received: April 24, 2015 Accepted: May 21, 2015 \\ doi: http://dx.doi.org/10.3329/jemc.v5i3.24744
}

\begin{abstract}
Background: Rectal cancer is one of the most common cancers in Kashmir, India. The clinical course of patients treated with surgery alone has been characterized by a high death rate and also by the pain and disability associated with pelvic recurrence of the tumor. Adjuvant radiation combined with chemotherapy has been studied for prevention of such recurrences. We treat more than 200 rectal cancer patients annually at our center. Most of the patients registered at our center are those who have been already subjected to surgery at the peripheral hospitals. We studied role of 5-fluorouracil (5-FU) and calcium leucovorin concurrently with radiotherapy in Dukes' stage B2 and C and toxicities thereof in the adjuvant setting. Objective: To assess the outcome of concurrent chemoradiation in operated locally advanced treated cancer patients. Materials and Methods: In operated Dukes' B2 and C rectal cancer patients, we conducted a prospective non-randomized study comprising of 40 patients between 2012 and 2014. Patients were treated with two hours protracted infusion of calcium leucovorin $500 \mathrm{mg} / \mathrm{m}^{2}$ on day 1 followed by 5 -fluorouracil $500 \mathrm{mg} / \mathrm{m}^{2}$ on days 1 to 5 and repeated four weekly for total of six cycles. Radiotherapy of 45 Gray in 20 fractions was delivered concurrently with chemotherapy for first two cycles. Results: Combination of chemotherapy and radiotherapy in a concurrent setting appears to be more efficient in reducing local recurrence rates and improving survival than either modality alone. Toxicities with this schedule were mostly gastrointestinal mucositis, but no treatment interruption was needed. Conclusion: A combination of 5-fluorouracil and radiotherapy can be administered in operated locally advanced rectal cancer patients.
\end{abstract}

Key words: Rectal cancer; 5-fluorouracil; Adjuvant; External beam radiotherapy

J Enam Med Col 2015; 5(3): 139-144

\section{Introduction}

Rectal cancers have a high local recurrence rate ranging from 2.6 to $47 \%$, even after curative resection. ${ }^{1}$ Tumor recurrence has been seen to correlate with stage, the number of metastatic lymph nodes, meso-rectal tumor spread, mucinous histology, lympho-vascular invasion and the distance of distal margins of resection. ${ }^{2}$ Rectal tumors resected with close margins are especially prone to recur locally. Approximately 8\% of Dukes' (A), 25$30 \%$ of Dukes' (B) and nearly $50 \%$ of Dukes' (C) develop pelvic recurrence after surgical resection. ${ }^{3}$ The incidence of local recurrence of tumors with negative nodes and only microscopic extension through wall is

1. Lecturer, Department of Radiotherapy, Government Medical College, Srinagar, Kashmir-190010, India

2. Assistant Professor, Department of Medicine, Government Medical College, Srinagar, Kashmir-190010, India

3. Registrar, Department of Radiotherapy, Government Medical College, Srinagar, Kashmir-190010, India

4. Lecturer, Department of Medicine, Government Medical College, Srinagar, Kashmir-190010, India

5. Registrar, Academic, Government Medical College, Srinagar, Kashmir-190010, India

6. Epidemiologist, Directorate of Health Services, Kashmir, India

Correspondence Syed Arshad Mustafa, Email: kadrism@gmail.com 
$17 \%$ and it increases to $54 \%$ in tumors adherent to or invading adjacent organs and structures. ${ }^{4}$ The areas of greatest risk for tumor recurrence are the pre-sacral space, pelvic side walls and soft tissue anterior to rectum. Addition of adjuvant radiation has significantly decreased loco-regional recurrences but local failures are still posing a big challenge to the oncologists. Chemotherapy combined with radiation as a radiosensitizer has been studied at many centers. It is also being increasingly used to facilitate a sphincter saving procedure in low-lying cancers. An optimal combination of chemo-radiotherapy for rectal cancer does not exist. A critical review of the literature shows that the superiority of chemo-radiation over radiation alone is weak or lacking. There is a great need for more conclusive study designs and a more rational explanation of drug-radiation interaction prior to clinical testing. ${ }^{5}$ At our center we have been treating large number of patients with locally advanced rectal cancers with adjuvant chemotherapy and radiotherapy in a sequential manner but so far have not studied concurrent chemoradiation in our setting. To our knowledge, this study is the first of its kind in Jammu \& Kashmir state of India.

\section{Materials and Methods}

Between 2012 and 2014, forty operated Dukes' B2 and $C$ rectal cancer patients were recruited in this prospective nonrandomized study. All the patients studied in this trial had histological documentation of adenocarcinoma with computed tomography (CT) scan of abdomen and chest ruling out any lung or liver metastasis. All patients underwent a potentially curative resection with no gross or microscopic evidence of any residual disease (R0 resection). Concurrent treatment was started within six weeks after surgery conducted after a valid consent. The study was reviewed and approved by the ethical committee of our institution. Patients were started on two hours protracted infusion of calcium leucovorin $500 \mathrm{mg} / \mathrm{m}^{2}$ on day one followed by 5 -fluorouracil $500 \mathrm{mg} / \mathrm{m}^{2}$ as a protracted infusion for two hours. 5-fluorouracil was instituted for 5 days a week. The chemotherapy was continued for a total of six cycles at an interval of four weeks. Radiation therapy was delivered by Cobalt-60 teletherapy unit by standard parallel opposed portals to a mid plane dose of 45 Grays for 4 weeks (five fractions per week). During entire period of chemo-radiation patients were assessed for treatment toxicities. All treatment related toxicities were defined as per Radiotherapy and Oncology Group
(RTOG) toxicity criteria. ${ }^{6}$ Patients were reviewed monthly after completion of treatment for one year and three monthly thereafter.

They were followed with clinical examination including digital rectal examination, serial serum CEA levels, chest radiography and yearly magnetic resonance imaging of abdomen and pelvis.

\section{Results}

Only 40 patients were eligible as per the predefined inclusion criteria which included pathological stages American Joint Committee on Cancer (AJCC) IIB and III, Eastern Cooperative Oncology Group (ECOG) performance score of less than or equal to 2, normal hemogram, liver function tests, kidney function tests, surgery done within six weeks, written informed consent from the patient, no significant associated comorbidity, no evidence of distant metastasis as defined by a CT scan of chest and abdomen, and patients not having received prior chemotherapy or radiotherapy. Majority of the patients $(52.5 \%)$ were aged less than or equal to 50 years with a male preponderance (male to female ratio of 1.35:1) (Table I). Most common presenting symptoms of patients were bleeding per rectum (80\%) and constipation (45\%). Thirty one $(77 \%)$ patients had growth located more than $5 \mathrm{~cm}$ above the anal verge. Low anterior resection was performed in $20(50 \%)$ patients and abdominoperineal resection was done in 17 (42\%). Transrectal excision was performed in only $3(7 \%)$ cases.

Table I: Patient characteristics $(\mathrm{N}=40)$

\begin{tabular}{|l|c|c|}
\hline \multirow{3}{*}{ Age } & $\leqslant 50$ & 21 \\
\hline \multirow{3}{*}{ Sex } & $>50$ & 19 \\
\hline & Males & 23 \\
\hline \multirow{3}{*}{ Stage (AJCC) } & Females & 17 \\
\hline & II B & 6 \\
\hline \multirow{3}{*}{ Surgical intervention } & III A & 10 \\
& III B & 19 \\
\hline \multirow{3}{*}{ Types of lesions on DRE } & III C & 5 \\
\hline \multirow{3}{*}{ HPE differentiation } & LAR & 20 \\
\hline & Transrectal & 17 \\
\hline & Polypoid & 21 \\
\hline & Ulcerative & 7 \\
\hline & WD & 27 \\
\hline
\end{tabular}


Since all patients were operated, accurate histopathological staging was available along with histopathological differentiation. Most of the patients $(28,70 \%)$ had well differentiated cancers on histology. Moderately differentiated and poorly differentiated tumors were found in $10(25 \%)$ and $2(5 \%)$ patients respectively. Staging was done as per AJCC. Thirty four $(85 \%)$ patients were having node positive disease (stage III), $6(15 \%)$ patients had stage II disease (Table I). In nine (23\%) patients disease was within $5 \mathrm{~cm}$ of anal verge, and in the rest $31(77 \%)$ the disease was high up. Low anterior resection was the procedure performed in $20(50 \%)$ cases, abdominoperineal resection (APR) in 17 (42.5\%) and transrectal excision in 3 $(7.5 \%)$ patients.

Treatment was well tolerated by majority of patients. In 5 patients treatment was interrupted because of toxicity (3, lower GI; 2, cardiac). Six patients required dose modification. Lower gastrointestinal toxicity (Table II) in the form of diarrhea was the commonest toxicity related symptom which was managed conservatively by antidiarrheals, antispasmodics and steroids. During chemoradiotherapy, asymptomatic bradycardia was unexpectedly found in 15 (37.5\%), while 2 patients developed chest pain. All these patients were followed with extensive cardiac monitoring including troponin $\mathrm{T}$ test and echocardiography to rule out any myocardial ischemia or cardiac dysfunction. In all patients bradycardia was reversible after chemotherapy was stopped and thus the treatment schedule was completed without any interruption. In all patients, 12 events of febrile neutropenia after chemotherapy were recorded. All these patients were admitted in indoor hospital wards and were managed successfully with antibiotics and other supportive measures.

Table II: Toxicity with the treatment schedule

\begin{tabular}{|c|c|c|c|}
\hline & Toxicity & Number & $\%$ \\
\hline \multirow[t]{2}{*}{ Mucosal toxicity } & Mucositis grade 3 & 5 & 12.5 \\
\hline & Mucositis grade 4 & 3 & 7.5 \\
\hline \multirow[t]{2}{*}{ Cardiac toxicity } & Asymptomatic bradycardia & 15 & 37.5 \\
\hline & Chest pain & 2 & 5 \\
\hline \multirow[t]{3}{*}{ Hematological toxicity } & Febrile neutropenia & 12 & 37.8 \\
\hline & Anemia & 12 & 54.1 \\
\hline & Thrombocytopenia & 03 & 8.1 \\
\hline \multirow[t]{3}{*}{ Cutaneous toxicity } & Skin pigmentation & 04 & 10.8 \\
\hline & Onycholysis & 03 & 8.1 \\
\hline & None & 30 & 81.1 \\
\hline
\end{tabular}

During a follow-up of 3 years $22(55 \%)$ patients have been disease free, 12 patients (30\%) relapsed (locoregional, 7 and distant, 5). Out of these, 4 patients recurred at local anastomotic site or perirectal area and 3 recurred in regional nodal areas. Lung ( 2 cases) and liver ( 2 cases) were the only sites of relapse among distant sites. In one patient, recurrence was found at both nodes and liver. After completion of treatment, 6 patients were lost to follow-up and could not be traced for evaluation.

Table III: Survival data (3-year follow-up)

\begin{tabular}{|l|c|c|}
\hline $\begin{array}{l}\text { Parameters } \\
\text { Relapse free }\end{array}$ & Number \\
\hline Relapse (12) & Local \\
\hline & Nodal & 4 \\
\hline & Lung & 3 \\
\hline Liver & 2 \\
\hline Died & Liver and Nodal & 2 \\
\hline Disease related & & 1 \\
\hline Disease unrelated & & 8 \\
\hline Lost to follow-up & & 6 \\
\hline
\end{tabular}

During follow-up, eight (20\%) patients were recorded dead in our study. Six $(15 \%)$ patients died of disease (2; locoregional, 3 ; distant failure; 1 ; local and distant metastasis) and one patient of the complications of diabetes mellitus type 2. Nodal metastasis was a significant predictor of both locoregional as well as distant failures.

\section{Discussion}

The management of rectal cancer has evolved from surgery alone to surgery with adjuvant and neoadjuvant treatment to present-day targeted therapies. Significant advances have been made in the study of rectal cancer over the last two decades and a more thorough understanding of the molecular basis of this disease, coupled with the development of new therapeutic approaches has dramatically altered the way in which patients are managed. ${ }^{7}$ Although, surgery is the treatment of choice for rectal cancer, local recurrence is common even after apparently curative resection. ${ }^{8}$ Clinical approach at adjuvant therapy for rectal cancer began more than 35 years ago and has involved numerous trials enrolling several thousand patients treated with 
cytotoxic drugs, non-specific immune stimulants to various combination thereof. ${ }^{9}$ Addition of chemotherapy to radiation is intended to decrease local recurrence and meanwhile control distant relapses. In North Central Cancer Treatment Group (NCCTG) trial $^{10}$, there was a $46 \%$ reduction in the pelvic recurrence, a $37 \%$ reduction in distant tumor spread and $29 \%$ reduction in patient deaths and the study confirmed the benefit achieved with chemotherapy when combined with irradiation. In our study after a follow-up of 3 years, 22 (55\%) patients were diseasefree which is comparable to most studies using concurrent adjuvant chemoradiation. In 12 patients, relapse was recorded (Table III) and most recurrences were locoregional (locoregional, 7; distant, 4 and locoregional with distant metastasis or spread, 1). None of the patients in the study group had undergone total mesorectal excision which could be the possible explanation for comparatively higher loco-regional failure rate.

A study was conducted by Conell \& co-workers in 1986 to determine the outcome of protracted venous infusion (PVI) of 5-fluorouracil concurrent with radiation vis-àvis bolus 5-fluorouracil and radiation with a median follow-up of 46 months of 686 patients with Dukes' B and $\mathrm{C}$ stage operated rectal carcinoma patients, those who received PVI during pelvic radiation had significantly increased time to relapse $(\mathrm{p}=0.01)$ and improved survival $(\mathrm{p}=0.005){ }^{11}$ Although radiation therapy decreased local recurrence in one half of patients, it was the addition of 5-fluorouracil based chemotherapy that further decreased local recurrence to approximately $10-12 \%$ and is the agent responsible for increasing overall 5 years survival rates by about $10-15 \% .^{12}$

In a randomized trial involving 144 cases in Norway, patients were randomized to postoperative radiation plus bolus 5-fluorouracil $\left(500 \mathrm{mg} / \mathrm{m}^{2}\right)$ day $1-2$ of week 1,2 and 3 of radiation versus surgery alone. Despite the fact that 5-fluorouracil was delivered with a radiosensitizing dose rather than dose adequate to treat systemic disease this combined modality therapy regimen significantly decreased local recurrence by $18 \%(\mathrm{p}=0.001)$ and improved 5 -year survival by $14 \% .^{13}$ Although the results with limited doses of 5fluorouracil are encouraging, additional experience with this approach is needed before modifying standard regimen.
In a Gastrointestinal Tumor Study Group (GITSG) trial, 227 patients with stage B2 and $\mathrm{C}$ rectal cancers were randomly allocated to receive: (i) no postoperative treatment, (ii) chemotherapy alone, (iii) pelvic radiotherapy or (iv) combined chemotherapy and radiation. After a median follow-up in excess of 10 years, the combined modality was found superior to surgery alone in terms of disease-free survival $(65 \%$ versus $45 \%$; $\mathrm{p}=0.006)$ and overall survival (45\% versus $26 \% ; \mathrm{p}=0.04) .{ }^{14}$ Although patients given combined modality had a greater likelihood of remaining free of recurrence than those treated with radiation alone or chemotherapy alone, these differences were not statistically significant.

The superiority of chemo-radiotherapy over radiation alone was confirmed in a subsequent study by the North Central Cancer Treatment Group which randomly assigned 240 patients with stages B2 or C rectal carcinoma to receive either postoperative radiotherapy alone (45Gy) or combined chemotherapy and radiation. After a median follow-up in excess of seven years the combined modality therapy was clearly superior, reducing the overall probability of recurrence rate by $46 \%$, the likelihood of developing distant metastasis by $37 \%(\mathrm{P}=0.04)$ and the mortality rate by $29 \%$ $(\mathrm{p}=0.003) .{ }^{14}$

Post-operative chemo-radiotherapy was the recommended standard therapy for patients with locally advanced rectal cancer. ${ }^{15}$ In a randomized study conducted by Sauer and co-workers to assess the overall regimen visa-vis pre-operative and post-operative adjuvant treatment in rectal cancer, 421 patients were randomly assigned to receive pre-operative chemo-radiotherapy and 402 patients to receive post-operative chemoradiotherapy. The overall 5-year survival rates were $76 \%$ and $74 \%$ respectively; cumulative incidence of local relapse was 6 percent for patients assigned to preoperative chemo-radiotherapy and $13 \%$ in postoperative chemo-radiotherapy group. Grade III and IV acute toxic effects occurred in 27 patients in the preoperative treatment group as compared with $40 \%$ of the patients in the post-operative treatment group; the corresponding rates of long term toxic effect were $14 \%$ and $24 \%$ respectively $(\mathrm{p}=0.0001)$. Pre-operative chemoradiotherapy as compared with post-operative chemoradiotherapy improved local control and was associated with reduced toxicity but did not improve overall survival. ${ }^{16}$ 
A study was conducted by $\mathrm{E}$ Krook et al ${ }^{10}$ to optimize the contribution of chemotherapy, decrease the local recurrence and improve survival with adjuvant radiation alone. Two hundred and four patients (with rectal cancer that was either locally advanced or metastatic to regional nodes) were randomly assigned to postoperative radiation alone or to radiation plus 5fluorouracil - both were preceded and followed by a cycle of systematic therapy with 5-fluorouracil plus methylchloro-ethylcyclohexylnitrosourea (MeCCNU). After a median follow-up of more than seven years, the combined modality treatment had overall recurrence rate reduced by $34 \%$, local recurrence was reduced by $46 \%$ and distant metastasis by $37 \%$. In addition, combined therapy reduced the rate of cancer related deaths by $36 \%$. Its acute toxic effects included nausea, vomiting, diarrhea, loss of appetite whereas delayed treatment related reactions like small bowel obstruction requiring surgery occurred in $6.7 \%$ of all patients receiving radiation and the frequencies of their complications were comparable in both treatment groups. The combination of post-operative therapy with radiation plus a 5-fluorouracil based regimen significantly and substantially improves the results in rectal carcinoma patients as compared to post-operative radiation alone. ${ }^{10}$

A randomized study was conducted by Fisher et $\mathrm{al}^{15}$ to assess the benefits of adjuvant chemotherapy and radiotherapy in operated rectal cancer patients. Patients were randomized to receive any of three measuresradiotherapy, chemotherapy or no further treatment. Their average time of study was 64.1 months. The chemotherapy groups when compared with the group treated by surgery alone demonstrated an overall improvement in disease-free survival $(\mathrm{p}=0.006)$ and in overall survival $(\mathrm{p}=0.08)$. When the group receiving post-operative radiation was compared to the group treated only by surgery, there was an overall reduction in locoregional recurrence from $25 \%$ to $16 \%$. No significant benefit in overall disease-free survival or survival from the use of radiation was demonstrated. In conclusion, the study demonstrated a benefit of adding adjuvant chemotherapy in the treatment of operated rectal cancer; post-operative radiation therapy although reduced the incidence of locoregional recurrence but it failed to affect overall disease-free survival. ${ }^{15}$

In yet another study Mimsky and co-workers compared the combined chemo-radiotherapy regimens of two separate parallel phase I trials to determine if combined pelvic radiotherapy, 5-fluorouracil and high dose leucovorin had less toxicity when delivered preoperatively versus post-operatively in patients with rectal cancer. In this study patients with unresectable disease received pre-operative radiotherapy plus 5fluorouracil and leucovorin followed by surgery and post-operative leucovorin and 5-fluorouracil. Patients with resectable disease received identical doses, techniques and schedules of radiotherapy and leucovorin and 5-fluorouracil except that the treatment was delivered post-operatively. Although more patients in the pre-operative group ( $75 \%$ versus $32 \%$; $=0.02$ ) received the higher doses level of 5 -fluorouracil (250 $\mathrm{mg} / \mathrm{m}^{2}$ ), significantly fewer experienced acute grade 3-4 toxicity ( $13 \%$ versus $48 \%$; $\mathrm{p}=0.045$ ). There was no grade 3-4 myelosuppression in either group. The two grade 3 toxicities in the pre-operative group were gastrointestinal. The grade 3 toxicities in the postoperative group included severe gastrointestinal and genitourinary symptoms; ${ }^{4}$ patients had grade 4 toxicity. Due to the high incidence of grade 3 and 4 toxicities reported in the post-operative combined modality group exploration of preoperative modality was suggested. ${ }^{17}$

In a randomized phase III trial by Sauer and co-workers to assess the efficacy of pre-operative versus postoperative radio-chemotherapy as regards to toxicity and post-operative morbidity patients with locally advanced operable rectal cancer were randomly assigned to preor postoperative radio-chemotherapy. The toxicity profile in both the groups was comparable. Postoperative complication rates were similar in both arms with $12 \%$ (post-operative radio-chemotherapy) and $13 \%$ (pre-operative radio-chemotherapy). The results of this trial were reported to be satisfactory and neo-adjuvant radio-chemotherapy was well tolerated and there was no higher risk as far as post-operative morbidity was concerned. ${ }^{18}$

From the findings of our study we can conclude that as a reference treatment a combination of 5-fluorouracil and radiotherapy can be advocated in locally advanced rectal cancer patients who have been subjected to surgery; but the mode of administration of 5-FU whether bolus or protracted infusion warrants further study. 


\section{References}

1. Garfinkel L, Mushinski M. U.S. cancer incidence mortality and survival, 1973-1996. Statistical Bulletin 1999; 80: 23.

2. Dukes CE, Burrey HJ. Spread of rectal cancer and its prognosis. British J Cancer 1958; 12: 309.

3. Khankbanian N, Mauglight GM, Russell WO, Schimek M. Significance of vascular invasion in colorectal cancer of Dukes `B`class. Cancer 1977; 39: 1192.

4. Rich T, Gynderson LL, Lew R, Galdibini JJ, Cohen AM, Donaldson G. Patterns of recurrence of rectal cancer after potentially curative surgery. Cancer 1983; 52: 1317.

5. Glimelius B. Chemo-radiotherapy in rectal cancer. Is there an optimal combination? Annals of Oncology 2001; 12(08): 1039-1045.

6. Cox JD, Stetz J, Pajak TF. Toxicity criteria of the Radiation Therapy Oncology Group (RTOG) and the European Organization for Research and Treatment of Cancer (EORTC). Int J Radiat Oncol Biol Phys 1995; 31(5): 1341-1346.

7. Carrato A. Adjuvant treatment of colorectal cancer. Gastrointest Cancer Res 2008; 2(4 Suppl 2): S42-S46.

8. Mc Dermott FT, Huges ESR, Pihl E, Johnson WR, Price AB. Local recurrence after potentially curative resection of rectal cancer. British Journal of Surgery 1985; 72: 34-37.

9. Windle R, Bell PRF, Shaw D. Five year results of a randomised trial of adjuvant 5-fluorouracil and levamisole in colorectal cancer. British Journal of Surgery 1987; 74(7): 569-572.

10. Krook J, Mooertal C, Gunderson L, Wieand HS, Roger TC, Beart RW et al. Effective surgical adjuvant therapy for high risk rectal carcinoma. N Engl J Med 1991; 324: 709-715.
11. MJO Conell, Martenson JA, Wieand HS, Krook J, MacDonald JS, Daniel G et al. Comparison of bolus versus protracted 5 Fluorouracil infusion in rectal cancer chemotherapy. N Engl J Med 1994; 331: 502-507.

12. Rosenberg SA. Decrease in local recurrence with addition of 5-Fluorouracil as adjuvant with external radiotherapy in operated rectal cancer. Cancer 2008; 13: 1296-1297.

13. Tveit KM, Guldvog I, Hagen S, Trondsen E, Harbitz T, Nygaard $\mathrm{K}$ et al. Impact of 5 Fluorouracil as radiosensitizer in decreasing local recurrence in operated rectal cancer. British Journal of Surgery 1997; 84: 1130-1135.

14. Gastrointestinal Tumor Study Group. Prolongation of disease free survival in surgically treated patients. N Eng J Med 1985; 312(23): 1465-1472.

15. Fisher B, Wolmark N, Rockette H, Redmond C, Deutsch M, Wickerham DL et al. Post operative adjuvant chemotherapy or radiotherapy for rectal cancer. J. Natl. Cancer Inst. 1988; 80: 21 .

16. Sauer R, Becker H, Hohenberger W, Rodel C, Wittekind C, Fietkau R et al. Pre operative versus post operative chemotherapy for rectal cancer. N Eng J Med 2004; 351: 1731-1740.

17. Mimsky BD, Cohen AM, Kemeny N, Enker WE, Kelsen DP, Reichman B et al. Combined modality therapy for rectal cancer. Decreased toxicity with pre-operative approach. Clinical Oncology 1992; 10: 1218-1224.

18. Sauer R, Fietkau R, Wittekind C, Martus P, Rodel C, Hohenberger $\mathrm{W}$ et al. Adjuvant versus neo adjuvant chemo radiotherapy for locally advanced rectal cancer. Oncology 2001; 177: 173-181. 